\title{
Differential gene expression between primary and secondary hyperparathyroidism
}

Shih-Ping Cheng, ${ }^{*}$ Chien-Liang Liu, ${ }^{*}$ Jie-Jen Lee, ${ }^{*}$ Han-Hsiang Chen, ${ }^{+}$Chih-Jen Wu ${ }^{\dagger}$

Departments of Surgery* and Nephrology ${ }^{\dagger}$, MacKay Memorial Hospital and Mackay Medical College, Taipei, Taiwan

\section{OBJECTIVES}

The pathophysiology differs significantly between primary hyperparathyroidism (pHPT) and secondary hyperparathyroidism (sHPT). The underling mechanisms of the occurrence of sporadic pHPT remain largely unknown. In this study, we analyzed the differences in gene expression between $\mathrm{pHPT}$ and $\mathrm{sHPT}$ to explore potential functional alterations.

\section{METHODS}

Total RNA was extracted from tissues obtained during parathyroidectomy. Gene expression microarray were performed. Pathways involving differential gene expressions were analyzed using the Kyoto Encyclopedia of Genes and Genomes (KEGG) database for functional enrichment.

\section{RESULTS}

Parathyroid tissues were classified in an unsupervised manner into primary and secondary clusters. A total of 311 genes were upregulated in pHPT, whereas 1358 genes were upregulated in sHPT. The expression of VEGF-A was significantly upregulated in $\mathrm{pHPT}$. The expression of transcription factor Twist was higher in SHPT.

\begin{tabular}{|c|c|c|c|c|}
\hline \multicolumn{5}{|c|}{ UPREGULATION in pHPT } \\
\hline Pathway & Count & $\%$ & $P$ value & Genes \\
\hline $\begin{array}{l}\text { Cell adhesion } \\
\text { molecules (CAMs) }\end{array}$ & 10 & 2.8 & 0.00091 & $\begin{array}{l}\text { NRCAM, ALCAM, } \\
\text { SELL, PVRL3, HLA- } \\
\text { A, NFASC, CLDN10, } \\
\text { HLA-C, HLA-B, } \\
\text { CD40, CD28 }\end{array}$ \\
\hline Allograft rejection & 4 & 1.1 & 0.031 & $\begin{array}{l}\text { HLA-A, HLA-C, } \\
\text { HLA-B, CD } 40, \text { CD } 28\end{array}$ \\
\hline $\begin{array}{l}\text { Chronic myeloid } \\
\text { leukemia }\end{array}$ & 5 & 1.4 & 0.056 & $\begin{array}{l}\text { E2F1, CDKN2A, } \\
\text { NFKBIA, MECOM, } \\
\text { SHC2 }\end{array}$ \\
\hline Nitrogen metabolism & 3 & 0.8 & 0.072 & CA4, CA2, CPS1 \\
\hline $\begin{array}{l}\text { Autoimmune thyroid } \\
\text { disease }\end{array}$ & 4 & 1.1 & 0.074 & $\begin{array}{l}\text { HLA-A, HLA-C, } \\
\text { HLA-B, CD } 40, \text { CD } 28\end{array}$ \\
\hline
\end{tabular}

\section{CONCLUSION}

Previous studies on hyperparathyroidism have focused on mutations in CASR, MEN1, RET, and HRPT2. Our study for the first time demonstrates that different pathophysiology led to differential gene profiling in hyperparathyroidism. It is particularly worth noting that parathyroid adenomas showed higher expression of cell adhesion molecules. On the contrary, secondary hyperparathyroidism exhibited upregulation of complement/coagulation cascades and mTOR signaling pathway.

\section{UPREGULATION in SHPT}

\begin{tabular}{|c|c|c|c|c|}
\hline Pathway & Count & $\%$ & $P$ value & Genes \\
\hline Ribosome & 30 & 2.7 & $2.1 \mathrm{E}-15$ & $\begin{array}{l}\text { RPL17, RPL15, RPL35, } \\
\text { RPS15A, RPS27L, } \\
\text { RPL39, RPS2, RPS3, } \\
\text { RPS29, RPL6, RPLP0, } \\
\text { RPLP1, RPL10, RPL5, } \\
\text { RPL11, RPL4, RSL24D1, } \\
\text { RPL7A, RPL10A, RPL12 } \\
\text { RPS24, RPL26, RPL28, } \\
\text { RPS8, RPL29, RPS18, } \\
\text { RPL22, RPS17, RPL21, } \\
\text { RPS13 }\end{array}$ \\
\hline $\begin{array}{l}\text { Intestinal } \\
\text { immune network } \\
\text { for IgA } \\
\text { production }\end{array}$ & 8 & 0.7 & 0.022 & $\begin{array}{l}\text { HLA-DQB1, CCL25, } \\
\text { CD40LG, TNFRSF13B, } \\
\text { IL15RA, HLA-DRB5, } \\
\text { HLA-DPB1, ITGA4 }\end{array}$ \\
\hline $\begin{array}{l}\text { Complement and } \\
\text { coagulation } \\
\text { cascades }\end{array}$ & 9 & 0.8 & 0.046 & $\begin{array}{l}\text { C8B, MBL2, F12, C4B, } \\
\text { TFPI, C1R, CFI, PROS1, } \\
\text { PLG }\end{array}$ \\
\hline $\begin{array}{l}\text { Hematopoietic } \\
\text { cell lineage }\end{array}$ & 10 & 0.9 & 0.062 & $\begin{array}{l}\text { IL3, IL9R, IL2RA, } \\
\text { DNTT, FLT3, CD2, } \\
\text { HLA-DRB5, ITGB3, } \\
\text { ITGA4, IL11RA }\end{array}$ \\
\hline $\begin{array}{l}\text { mTOR signaling } \\
\text { pathway }\end{array}$ & 7 & 0.6 & 0.081 & $\begin{array}{l}\text { EIF4B, RPS6KA1, ULK2 } \\
\text { RPS6KB2, IGF1, IGF2, } \\
\text { FIGF }\end{array}$ \\
\hline
\end{tabular}

\section{REFERENCES}

1. Cheng SP et al. Association between parathyroid hormone levels and inflammatory markers among US adults. Mediators Inflamm 2014;2014:709024.

2. Cheng SP et al. Gender differences among patients with secondary hyperparathyroidism undergoing parathyroidectomy. J Surg Res 2011;168:82-7.

3. Cromer MK et al. Identification of somatic mutations in parathyroid tumors using whole-exome sequencing. $J$ Clin Endocrinol Metab 2012;97:E1774-81. 